\title{
Electroconvulsive Therapy on Treatment-resistant Mania in Bipolar Disorder with No Concurrent Antipsychotics: A Case Report
}

\author{
Takako Enokida, Takamasa Noda, Takashi Usami, Naoko Satake, Kazuyuki Nakagome \\ Department of Psychiatry, National Center of Neurology and Psychiatry, Tokyo, Japan
}

Pharmacotherapy is generally the first choice for the treatment of acute mania in bipolar disorder. Electroconvulsive therapy (ECT) is reported to be an effective treatment modality for mania; however, it is usually used as the "last resort." Herein, we report a case of a patient with treatment-resistant severe mania in bipolar disorder who recovered with ECT without concurrent antipsychotics and mood stabilizers. Our case report showed that ECT monotherapy can be an effective treatment modality for manic state in bipolar disorder, which may lead to a shorter hospital stay and better social outcomes.

KEY WORDS: Mania; Bipolar disorder; Electroconvulsive therapy; Treatment-resistant.

\section{INTRODUCTION}

Severe mania presents with various symptoms, such as suicidal or homicidal ideation, aggressiveness, psychosis, and other symptoms, and is an urgent medical condition. Pharmacotherapy is generally the first choice for the treatment of acute mania in bipolar disorder, and lithium, anticonvulsants, and antipsychotics are the mainstay of pharmacotherapy [1-3]. Several meta-analyses on severe mania have reported that the combination of lithium or valproate with antipsychotics is more efficacious than the monotherapy of mood stabilizers [4]. In clinical practice, mood stabilizers and antipsychotics are often used in combination.

Electroconvulsive therapy (ECT) is effective for manic state in bipolar disorder, and ECT should not be considered the "last resort" $[5,6]$. While randomized controlled trials comparing medication with ECT in mania have not been conducted, a previous report suggested that ECT showed marked improvement in bipolar disorder and

Received: January 4, 2021 / Accepted: February 15, 2021

Address for correspondence: Takamasa Noda

Department of Psychiatry, National Center of Neurology and Psychiatry, 4-1-1 Ogawahigasicho, Kodaira, Tokyo 1878551, Japan

E-mail: t-noda@ncnp.go.jp

ORCID: https://orcid.org/0000-0003-0655-9350 could be superior to pharmacotherapy [7]. However, in patients with manic state in bipolar disorder, ECT is advised to only those patients who are refractory and who do not respond to three to six combinations of medications $[1,8]$. Therefore, the patient in the manic state requires a longer time to undertake ECT. Only a few studies have reported the use of ECT in patients with mania without antipsychotics or mood stabilizers; however, these patients were not completely medication-free.

We, herein, report a patient with mania who underwent ECT without medications. To our knowledge, this is the first case report of the safe administration of ECT for mania with no concurrent medication. Informed consent was provided by the patient for this case report.

\section{CASE}

A 39-year-old woman was admitted to our hospital. Nineteen years prior, she was diagnosed with bipolar I disorder in a manic state and was admitted to another hospital. She was prescribed haloperidol; however, soon she developed fever and her creatine kinase (CK) levels were elevated. Malignant syndrome was suspected, and haloperidol was discontinued the day following the fever. She was treated with ECT on the same day. After four sessions of ECT, she had remission of symptoms and was dis-

@) This is an Open-Access article distributed under the terms of the Creative Commons Attribution Non-Commercial License (http://creativecommons.org/licenses/by-nc/4.0) which permits unrestricted non-commercial use, distribution, and reproduction in any medium, provided the original work is properly cited. 
charged from the hospital. She was prescribed lithium $500 \mathrm{mg}$ per day and sulpiride $150 \mathrm{mg}$ per day. Her prescription was tapered off, and she had been medicationfree. She had a final consultation 16 years ago, and was free of significant recurrence of mania or depression in the past 17 years.

She became depressed 2 years ago and became progressively agitated for 1 year. She had trouble of human relationships with colleagues and was finally unemployed (probably fired from her job). She was first admitted to a local hospital. On admission, she presented with agitation and persistent insomnia. On the first day of hospitalization, she made many calls to her coworkers and the fire-department. Aripiprazole $24 \mathrm{mg}$ per day was prescribed, and lithium $200 \mathrm{mg}$ per day was added. Since her manic symptoms did not improve, her prescription was switched from aripiprazole to olanzapine. However, coarse tremor and bilateral knee joint pain appeared. Laboratory data showed elevated CK levels to $384 \mathrm{IU} / \mathrm{L}$; hence, olanzapine and lithium were tapered off. Quetiapine 200 mg per day was initiated; however, coarse tremor, salivation, dysphagia, and CK elevation reappeared. Quetiapine was switched to zotepine; however, salivation and dysphagia reappeared, and hence, zotepine was subsequently tapered. Since it was difficult to control her symptoms by medication, she was transferred to our hospital for further therapy.

She had a past medical history of appendicitis and hyperthyroidism and was on propylthiouracil for 6 months. She had a family history of depression, her mother had depression and her younger sister had postpartum depression. The patient reported that she had never smoked, consumed $700 \mathrm{ml}$ of alcohol per day until before her previous admission, and denied any substance abuse.

On physical examination, she was exacerbated. Her height was $157 \mathrm{~cm}$, body weight was $36 \mathrm{~kg}$, and body mass index was $14.6 \mathrm{~kg} / \mathrm{m}^{2}$. Results of routine laboratory investigations revealed that CK levels were elevated to $995 \mathrm{IU} / \mathrm{L}$, and mild liver dysfunction was detected. Her thyroid hormone levels were within the normal range. Electrocardiography, chest and abdominal radiography, and head computed tomography revealed no abnormalities. Mental status examination revealed increased tone tempo and quantum of speech with flight of ideas, inflated selfesteem, irritability, and decreased need for sleep. The Japanese version of the Young Mania Rating Scale (YMRS-J) score on the first day of admission was $44 / 60$. She was on flunitrazepam $2 \mathrm{mg}$, zotepine $25 \mathrm{mg}$, triazolam $0.25 \mathrm{mg}$, mosapride citrate hydrate $15 \mathrm{mg}$, and magnesium oxide 1,980 mg per day.

We planned to perform bilateral ECT twice or thrice a week for a total of 12 sessions without mood stabilizers or antipsychotics, and then initiate lithium as a maintenance treatment after the completion of ECT. Flunitrazepam was reduced from 2 to $1 \mathrm{mg}$, and we excluded zotepine, mosapride citrate hydrate, and triazolam from the preparation for ECT. The patient underwent ECT on the third day of admission to our hospital. In the first session, generalized tonic/clonic seizures were induced, which lasted for approximately 125 seconds, and the patient recovered well. After six sessions, she became less irritated. Furthermore, her tone tempo and quantum of speech decreased. Her YMRS-J score decreased from 44 to $11 / 60$. After seven sessions, she slept well; hence, the dose of flunitrazepam was reduced to $0.5 \mathrm{mg}$ per day. Her YMRS-J score gradually decreased to 2/60 after 12 sessions. She was responsive in every session, and the mean seizure duration was 61.25 seconds. Lithium $200 \mathrm{mg}$ per day was started at the end of the ECT session. Laboratory data showed no abnormalities except for low thyroid-stimulating hormone (TSH) levels. We increased the dose of lithium from 200 to $400 \mathrm{mg}$ per day. She gradually became euphoric and emotionally unstable. Her YMRS-J score was slightly elevated to $11 / 60$. We increased the dose of lithium to 500 mg per day; however, she developed eczema on the face and low TSH levels persisted. She was assumed to have developed destructive thyroiditis caused by lithium. Her quantum of speech started to increase, and her YMRS-J score rapidly increased. We discontinued lithium and started the second term of ECT to treat her manic relapse. Flunitrazepam was terminated, and she was totally drugfree after three sessions. After five sessions, her quantum of speech markedly decreased, and she was in remission. We initiated valproate $400 \mathrm{mg}$ per day, and increased the dose up to $600 \mathrm{mg}$ after one week. Her valproate concentration level was 105 (normal range: 50-100), so we decreased the dose to $500 \mathrm{mg}$ per day. She had been in full remission and was subsequently discharged. She started a new job and has maintained remission for one and a half years. 


\section{DISCUSSION}

Our patient presented with severe symptoms of mania, such as aggressiveness, impaired judgment and decisionmaking abilities, and psychosis. Despite the severity of her symptoms, we were not able to administer a sufficient dose of antipsychotics because of the side effects, and adequate improvement was not achieved even after the administration of mood stabilizers. Nevertheless, we succeeded in controlling severe mania by ECT without medications. Valproate was used as the maintenance drug after ECT.

Lithium and valproate are usually used as initial treatments for mania; however, their effect has a time interval until response. In addition, antipsychotics have an immediate effect and are often used to sedate and rest manic patients $[1,2]$. Meta-analyses of 68 randomized trials reported that antipsychotics are more efficacious than mood stabilizers as in the evaluation of a 3-week manic state [9]. Some patients, especially those at risk of self-harm or in a state of high aggression, need immediate response to treatment to protect themselves and others. Severe mania often results in impaired psychosocial functioning, which makes it difficult for patients to maintain relationships in their community. Mania should be treated immediately from both the psychological and social prognosis viewpoints. Therefore, in the cases of poorly tolerated antipsychotics or mood stabilizers, and when mania is too fast to progress or relapse, the duration of hospitalization is prolonged

ECT provides a rapid clinical response. Nevertheless, the administration of ECT as first-line treatment is usually limited to patients with major depression or with severe psychosis. In bipolar disorder, ECT is not the first choice of treatment. ECT plus pharmacotherapy is indicated secondary for those who fail pharmacotherapy alone, and ECT is found to be effective $[6,10]$. A review of several case series reported that ECT is safe with lithium [11], but it is still controversial. No consensus regarding performing ECT in patients who are on mood stabilizers is available. In a case series reported in 2005, three manic patients were treated with ECT, one with antipsychotics, another with TSH, and the other with medications of Acquired immunodeficiency syndrome; however, these patients were not completely medication-free [12]. Our case shows that ECT alone can be selected for fast therapy in severe acute manic episodes. Early ECT treatment may lead to shorter hospitalization and better social outcomes for the patients.

Acute extrapyramidal syndrome is induced by antipsychotics owning to genetic factors [13]. In our case, the hypersensitivity to antipsychotics may be caused by gene polymorphisms or abnormal metabolizer status for cytochromes P450 family [14]. This is a limited consideration because we did not analyze the genetic polymorphism of the patient.

In conclusion, herein, we report a case of treatment-resistant severe mania in bipolar disorder, in which we had difficulties in prescribing antipsychotics or mood stabilizers because of complications. This case report shows that ECT monotherapy can be an effective treatment modality for mania.

Funding

None.

\section{Conflicts of Interest}

No potential conflict of interest relevant to this article was reported.

\section{Author Contributions}

Conceptualization: Takako Enokida, Takamasa Noda. Data acquisition: Takako Enokida. Supervision: Kazuyuki Nakagome. Writing-original draft: Takako Enokida. Writing-review \& editing: Takako Enokida, Takamasa Noda, Takashi Usami, Naoko Satake, Kazuyuki Nakagom.

\section{ORCID}

Takako Enokida https://orcid.org/0000-0002-7343-9300

Takamasa Noda https://orcid.org/0000-0003-0655-9350

Takashi Usami https://orcid.org/0000-0003-2286-8663

Naoko Satake https://orcid.org/0000-0003-3761-6629

Kazuyuki Nakagom https://orcid.org/0000-0003-2919-8180

\section{REFERENCES}

1. Grunze H, Vieta E, Goodwin GM, Bowden C, Licht RW, Azorin JM, et al. The World Federation of Societies of Biological Psychiatry (WFSBP) guidelines for the biological treatment of bipolar disorders: acute and long-term treatment of mixed states in bipolar disorder. World J Biol Psychiatry 2018;19:2-58.

2. Yatham LN, Kennedy SH, Parikh SV, Schaffer A, Bond DJ, Frey $\mathrm{BN}$, et al. Canadian Network for Mood and Anxiety Treatments (CANMAT) and International Society for Bipolar Disorders (ISBD) 2018 guidelines for the management of patients with bipolar disorder. Bipolar Disord 2018;20:97-170. 
3. Goodwin GM, Haddad PM, Ferrier IN, Aronson JK, Barnes T, Cipriani A, et al. Evidence-based guidelines for treating bipolar disorder: revised third edition recommendations from the British Association for Psychopharmacology. J Psychopharmacol 2016;30:495-553.

4. Malhi GS, Gessler D, Outhred T. The use of lithium for the treatment of bipolar disorder: recommendations from clinical practice guidelines. J Affect Disord 2017;217:266-280.

5. American Psychiatric Association. Task Force on Electroconvulsive Therapy. The practice of ECT: recommendations for treatment, training and privileging. Convuls Ther 1990;6:85120.

6. Perugi G, Medda P, Toni C, Mariani MG, Socci C, Mauri M. The role of Electroconvulsive Therapy (ECT) in Bipolar Disorder: effectiveness in 522 patients with bipolar depression, mixedstate, mania and catatonic features. Curr Neuropharmacol 2017:15:359-371.

7. Abrams R, Taylor MA. Unipolar and bipolar depressive illness. Phenomenology and response to electroconvulsive therapy. Arch Gen Psychiatry 1974;30:320-321.

8. Hilty DM, Brady KT, Hales RE. A review of bipolar disorder among adults. Psychiatr Serv 1999;50:201-213.

9. Cipriani A, Barbui C, Salanti G, Rendell J, Brown R, Stockton $\mathrm{S}$, et al. Comparative efficacy and acceptability of antimanic drugs in acute mania: a multiple-treatments meta-analysis. Lancet 2011;378:1306-1315.

10. Patel RS, Jain SB, Youssef NA. Electroconvulsive treatment utilization for the inpatient management of severe manic episodes of bipolar disorder. J ECT 2019;35:195-200.

11. Dolenc TJ, Rasmussen KG. The safety of electroconvulsive therapy and lithium in combination: a case series and review of the literature. J ECT 2005;21:165-170.

12. Macedo-Soares MB, Moreno RA, Rigonatti SP, Lafer B. Efficacy of electroconvulsive therapy in treatment-resistant bipolar disorder: a case series. J ECT 2005;21:31-34.

13. MacNeil RR, Müller DJ. Genetics of common antipsychoticinduced adverse effects. Mol Neuropsychiatry 2016;2:61-78.

14. Fleeman N, Dundar Y, Dickson R, Jorgensen A, Pushpakom S, McLeod C, et al. Cytochrome P450 testing for prescribing antipsychotics in adults with schizophrenia: systematic review and meta-analyses. Pharmacogenomics J 2011;11:1-14. 